\title{
Design of Epitope-Based Peptide Vaccine against Pseudomonas aeruginosa Fructose Bisphosphate Aldolase Protein Using Immunoinformatics
}

\author{
Mustafa Elhag $\mathbb{D}^{1}$ Ruaa Mohamed Alaagib $\mathbb{D}^{2}{ }^{2}$ Nagla Mohamed Ahmed $\left(\mathbb{D},{ }^{3}\right.$ \\ Mustafa Abubaker $\mathbb{D}^{4}{ }^{4}$ Esraa Musa Haroun ${ }^{D},{ }^{5}$ Sahar Obi Abd Albagi $\mathbb{D}^{3}{ }^{3}$ \\ and Mohammed A. Hassan ${ }^{6}{ }^{6}$ \\ ${ }^{1}$ Faculty of Medicine, University of Seychelles-American Institute of Medicine, Seychelles \\ ${ }^{2}$ Department of Pharmacies, National Medical Supplies Fund, Sudan \\ ${ }^{3}$ Faculty of Medical Laboratories Sciences, Al-Neelain University, Sudan \\ ${ }^{4}$ Faculty of Medical Laboratory Sciences, Sudan University of Science and Technology, Sudan \\ ${ }^{5}$ Faculty of Medical Pharmacology, Ahfad University for Women, Sudan \\ ${ }^{6}$ Department of Bioinformatics, DETAGEN Genetics Diagnostic Center, Kayseri, Turkey
}

Correspondence should be addressed to Mustafa Elhag; mustafa@comxtreme.com

Received 10 September 2019; Revised 21 September 2020; Accepted 6 October 2020; Published 6 November 2020

Academic Editor: Enrique Ortega

Copyright (C) 2020 Mustafa Elhag et al. This is an open access article distributed under the Creative Commons Attribution License, which permits unrestricted use, distribution, and reproduction in any medium, provided the original work is properly cited.

Pseudomonas aeruginosa is a common pathogen that is responsible for serious hospital-acquired infections, ventilator-associated pneumonia, and various sepsis syndromes. Also, it is a multidrug-resistant pathogen recognized for its ubiquity and its intrinsically advanced antibiotic-resistant mechanisms. It usually affects immunocompromised individuals but can also infect immunocompetent individuals. There is no vaccine against it available till now. This study predicts an effective epitope-based vaccine against fructose bisphosphate aldolase (FBA) of Pseudomonas aeruginosa using immunoinformatics tools. The protein sequences were obtained from NCBI, and prediction tests were undertaken to analyze possible epitopes for B and T cells. Three B cell epitopes passed the antigenicity, accessibility, and hydrophilicity tests. Six MHC I epitopes were found to be promising, while four MHC II epitopes were found promising from the result set. Nineteen epitopes were shared between MHC I and II results. For the population coverage, the epitopes covered $95.62 \%$ worldwide excluding certain MHC II alleles. We recommend in vivo and in vitro studies to prove its effectiveness.

\section{Introduction}

Pseudomonas aeruginosa is a motile, nonfermenting, gramnegative opportunistic bacterium that is implicated in respiratory infections, urinary tract infections, gastrointestinal infections, keratitis, otitis media, and bacteremia in patients with compromised host defences (e.g., cancer, burn, HIV, and cystic fibrosis) [1]. Intensive care unit (ICU) hospitalized patients constitute one of the risk groups that are more susceptible to acquire pseudomonas infections as they may develop ventilator-associated pneumonia (VAP) and sepsis [2-4]. This organism is a ubiquitous and metabolically versa- tile microbe that flourishes in many environments and possesses many virulence factors that contribute to its pathogenesis [1]. According to data from Centers for Disease Control, $P$. aeruginosa is responsible for millions of infections each year in the community, $10-15 \%$ of all healthcare-associated infections, with more than 300,000 cases annually in the EU, USA, and Japan [5]. It is a common nosocomial pathogen $[6,7]$ that causes infections with a high mortality rate $[8,9]$ which is attributable to the organism that possesses an intrinsic resistance to many antimicrobial agents [10] and the development of increased, multidrug resistance in healthcare settings [11-13], both of which 
Amino acid composition

gi|15595752|ref|NP_249246.1| fructose-1, 6-bisphosphate aldolase (Pseudomonas aeruginosa PAO1)

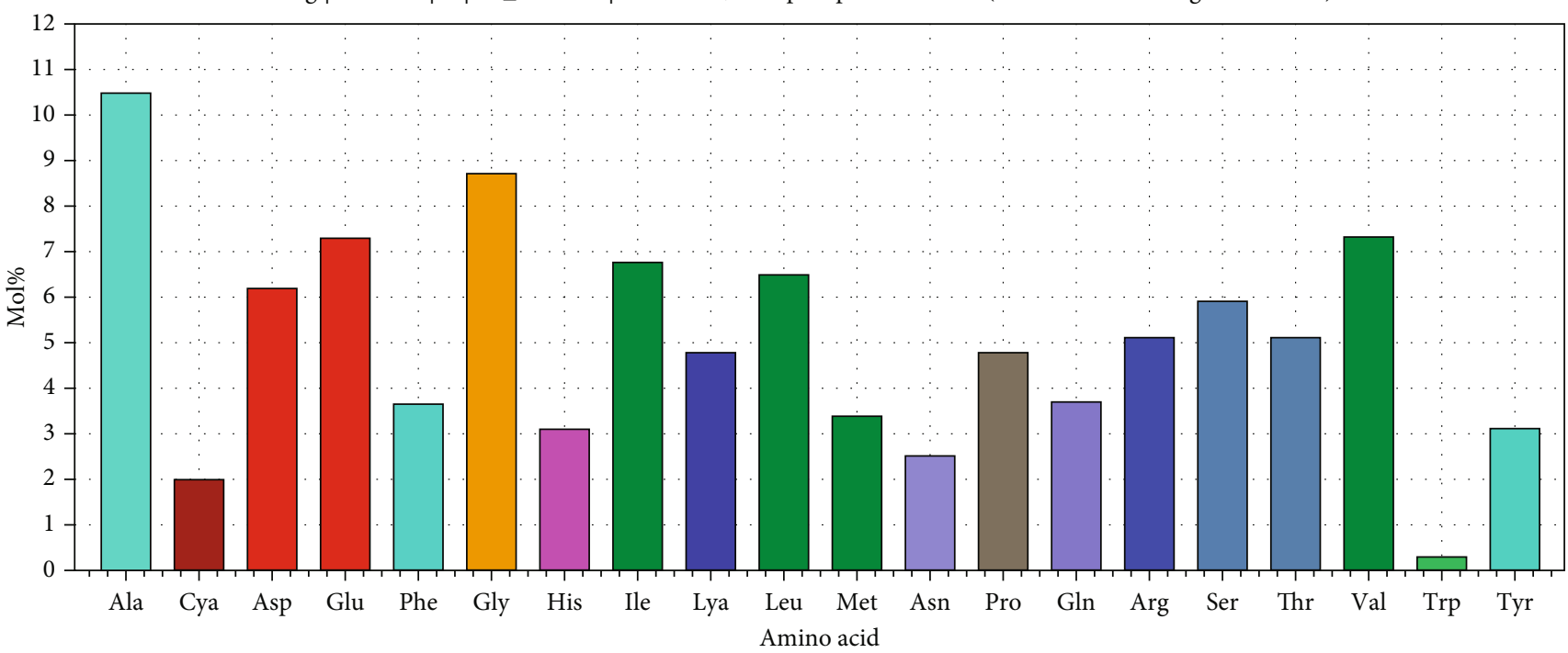

FIgURE 1: Amino acid composition for Schistosoma mansoni FBA using BioEdit software.

complicate antipseudomonal chemotherapy. As a result, it remains difficult to combat $P$. aeruginosa infections despite supportive treatments. Vaccines could be an alternative strategy to control $P$. aeruginosa infections and even reduce antibiotic resistance; however, no $P$. aeruginosa vaccine is currently available [14]. Döring and Pier represented that the serious obstacle to the development of a globally effective anti-P. aeruginosa vaccine is due to the antigenic variability of a microorganism that enables it to easily adapt to different growth conditions and escapes host immune recognition and to the high variability of the proteins among different $P$. aeruginosa strains and within the same strain, grown in diverse environmental conditions [15].

Contemporary, integrated genomics and proteomics approaches have been used to predict vaccine candidates against $P$. aeruginosa [16]. Although several vaccine formulations have been clinically tested, none has been licensed yet $[15,17]$. The search for new targets or vaccine candidates is of high paramount. Bioinformatics-based approach is a novel platform to identify drug targets and vaccine candidates in human pathogens $[18,19]$. Thus, the present study is aimed at designing an effective peptide vaccine against $P$. aeruginosa using computational approach through prediction of highly conserved $\mathrm{T}$ and $\mathrm{B}$ cell epitopes from the highly immunogenic protein fructose bisphosphate aldolase (FBA). This is the first study that predicts epitope-based vaccine from this moonlighting protein of $P$. aeruginosa. This technique has been successfully used by many authors to identify target vaccine candidates. These types of vaccines are easy to produce, specific, capable of keeping away any undesirable immune responses, reasonable, and safe when compared to the conventional vaccines such as killed and attenuated vaccines [20].

\section{Materials and Methods}

2.1. Protein Sequence Retrieval. A total of 20,201 strains of Pseudomonas aeruginosa FBA were retrieved in FASTA for-
TABLE 1: Molecular weight and amino acid frequency distribution of the protein.

\begin{tabular}{lcc}
\hline Amino acid & Number & Mol\% \\
\hline Ala & 37 & 10.45 \\
Cys & 4 & 1.13 \\
Asp & 22 & 6.21 \\
Glu & 26 & 7.34 \\
Phe & 13 & 3.67 \\
Gly & 31 & 8.76 \\
His & 11 & 3.11 \\
Ile & 24 & 6.78 \\
Lys & 17 & 4.8 \\
Leu & 23 & 6.5 \\
Met & 12 & 3.39 \\
Asn & 9 & 2.54 \\
Pro & 17 & 4.8 \\
Gln & 13 & 3.67 \\
Arg & 18 & 5.08 \\
Ser & 21 & 5.93 \\
Thr & 18 & 5.08 \\
Val & 26 & 7.34 \\
Trp & 1 & 0.28 \\
Tyr & 11 & 3.11 \\
\hline
\end{tabular}

mat from the National Center for Biotechnology Information (NCBI) database (https://ncbi.nlm.nih.gov) on May 2019. The protein sequence had a length of 354 with the name fructose-1,6-bisphosphate aldolase.

2.2. Determination of Conserved Regions. The retrieved sequences of Pseudomonas aeruginosa FBA were subjected to multiple sequence alignment (MSA) using the ClustalW 
Table 2: List of conserved peptides with their antigenicity, Emini surface accessibility, and Parker hydrophilicity scores ( ${ }^{*}$ peptides that successfully passed the three tests).

\begin{tabular}{|c|c|c|c|c|c|c|}
\hline Peptide & Start & End & Length & $\begin{array}{c}\text { Kolaskar \& Tongaonkar } \\
\text { antigenicity score } \\
\text { (TH: } 1.025)\end{array}$ & $\begin{array}{l}\text { Emini surface } \\
\text { accessibility } \\
\text { score (TH: } 1 \text { ) }\end{array}$ & $\begin{array}{c}\text { Parker hydrophilicity } \\
\text { prediction score } \\
\text { (TH: } 1.681)\end{array}$ \\
\hline RQMLDHAA & 7 & 14 & 8 & 1.008 & 1.013 & 1.637 \\
\hline FNVNNLEQMRAIM & 23 & 35 & 13 & 0.974 & 0.432 & 0.2 \\
\hline AADKTDSPVIVQASAGARK & 37 & 55 & 19 & 1.031 & 0.776 & 3.3 \\
\hline ADKTDSPVI* & 38 & 46 & 9 & 1.027 & 1.084 & 3.322 \\
\hline MHQDHGTSPDVCQ & 80 & 92 & 13 & 1.035 & 0.868 & 3.785 \\
\hline SIQLGFSSVMMDGSL & 94 & 108 & 15 & 1.03 & 0.082 & 0.38 \\
\hline EDGKTP & 110 & 115 & 6 & 0.916 & 3.211 & 6.083 \\
\hline YNVRVTQQTVA & 120 & 130 & 11 & 1.079 & 0.972 & 2.064 \\
\hline YNVRVTQQTV* & 120 & 129 & 10 & 1.081 & 1.229 & 2.06 \\
\hline AHACGVSVEGELGCLGSLETGM & 132 & 153 & 22 & 1.057 & 0.011 & 1.818 \\
\hline GEEDG & 155 & 159 & 5 & 0.863 & 1.532 & 7.4 \\
\hline GAEGVLDHSQ & 161 & 170 & 10 & 1.029 & 0.539 & 3.3 \\
\hline LTDPEE & 172 & 177 & 6 & 0.965 & 2.317 & 3.95 \\
\hline DALAIAIGTSHGAY & 188 & 201 & 14 & 1.044 & 0.109 & 1.179 \\
\hline THLVMHGSSSVPQ & 227 & 239 & 13 & 1.073 & 0.369 & 1.685 \\
\hline HGSSSVPQ* & 232 & 239 & 8 & 1.06 & 1.016 & 3.963 \\
\hline WLAII & 241 & 245 & 5 & 1.102 & 0.134 & -6.62 \\
\hline YGGEIKETYG & 248 & 257 & 10 & 0.964 & 1.408 & 3.3 \\
\hline KVNIDTDLRLAST & 273 & 285 & 13 & 1.018 & 0.843 & 1.985 \\
\hline AMRD & 311 & 314 & 4 & 0.907 & 1.279 & 3.025 \\
\hline GTAGN & 324 & 328 & 5 & 0.899 & 0.717 & 5.14 \\
\hline GEL & 347 & 349 & 3 & 0.992 & 0.694 & 1.433 \\
\hline
\end{tabular}

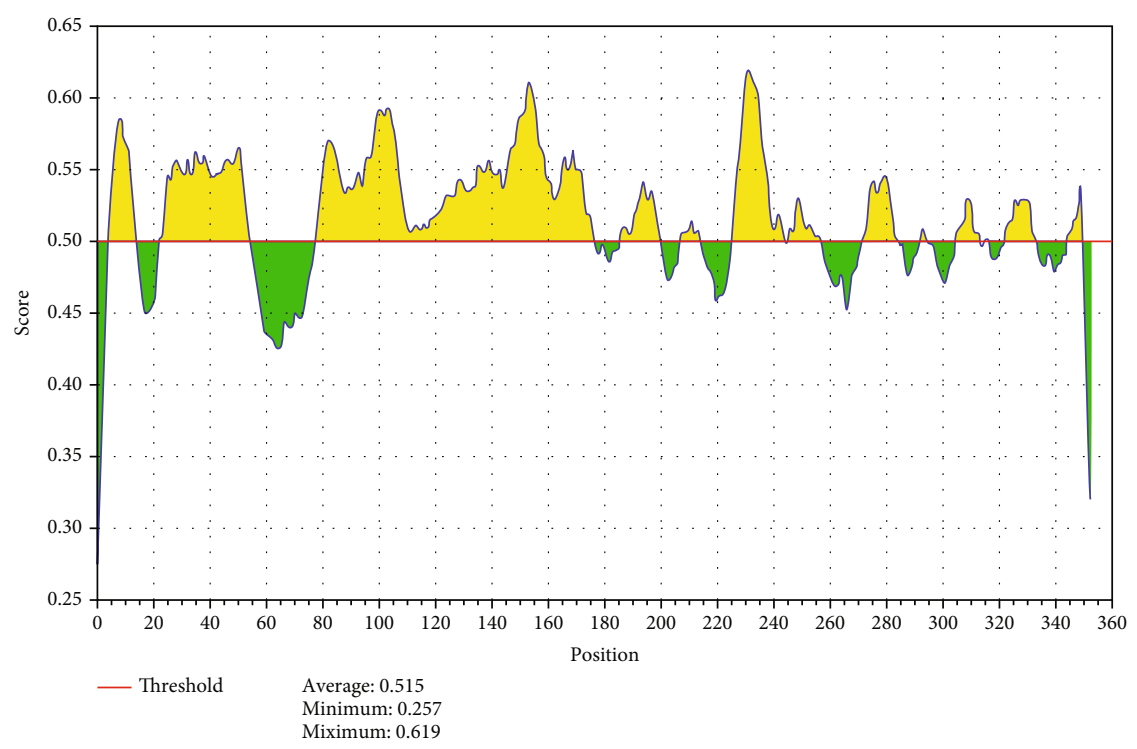

FIGURE 2: BepiPred linear epitope prediction; yellow areas above the threshold (red line) are proposed to be a part of B cell epitopes, and the green areas are not.

tool of BioEdit Sequence Alignment Editor Software version 7.2.5 to determine the conserved regions. Also, molecular weight and amino acid composition of the protein were obtained [21, 22].
2.3. Sequenced-Based Method. The reference sequence (NP 249246.1) of Pseudomonas aeruginosa FBA was submitted to different prediction tools at the Immune Epitope Database (IEDB) Analysis Resource (http://www.iedb.org/) to predict 


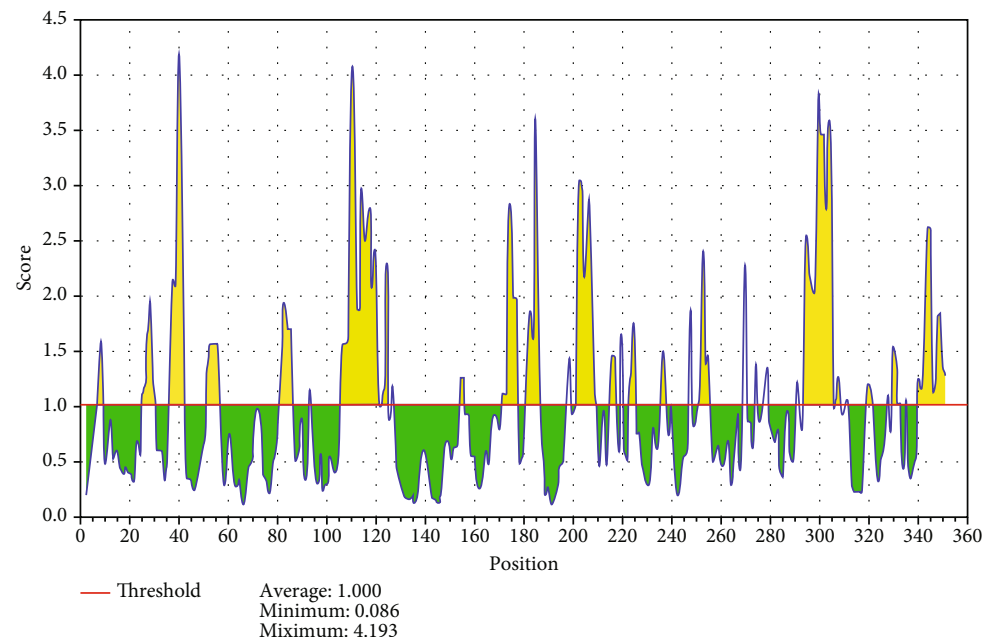

FIGURE 3: Emini surface accessibility prediction; yellow areas above the threshold (red line) are proposed to be a part of B cell epitopes, and the green areas are not.

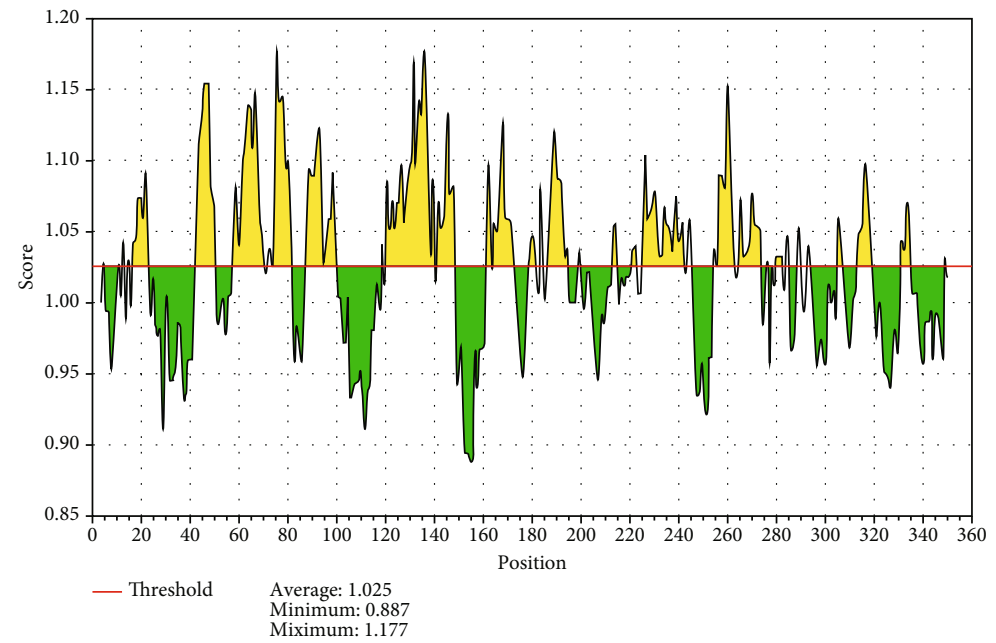

FIGURE 4: Kolaskar and Tongaonkar antigenicity prediction; yellow areas above the threshold (red line) are proposed to be a part of B cell epitopes, and green areas are not.

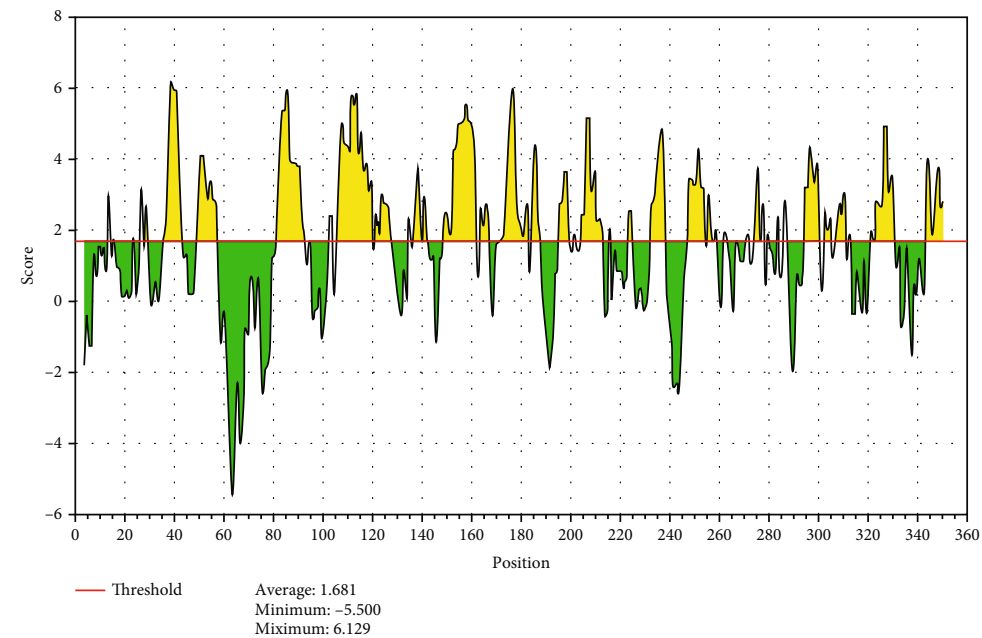

Figure 5: Parker hydrophilicity prediction; yellow areas above the threshold (red line) are proposed to be a part of B cell epitopes, and green areas are not. 

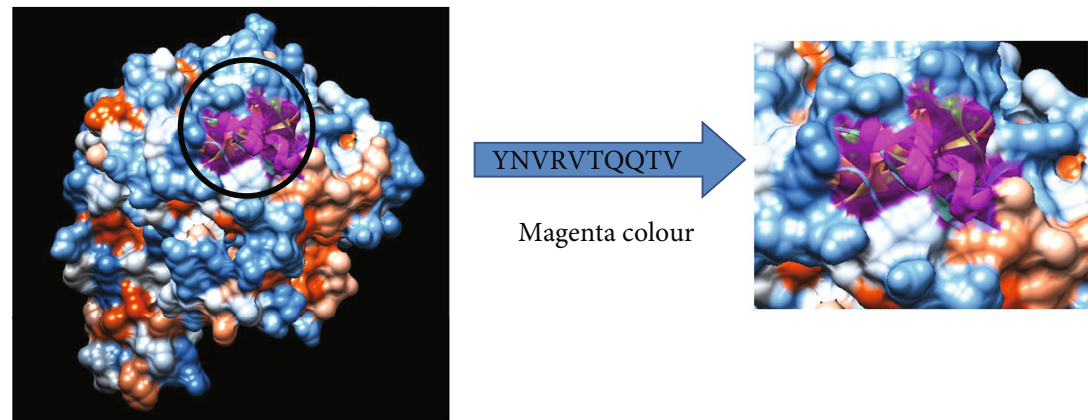

FIGURE 6: B cell epitopes proposed. The arrow shows the position of YNVRVTQQTV with Magenta colour in a structural level of fructose 1,6bisphosphate aldolase. *The 3D structure was obtained using USCF Chimera software.
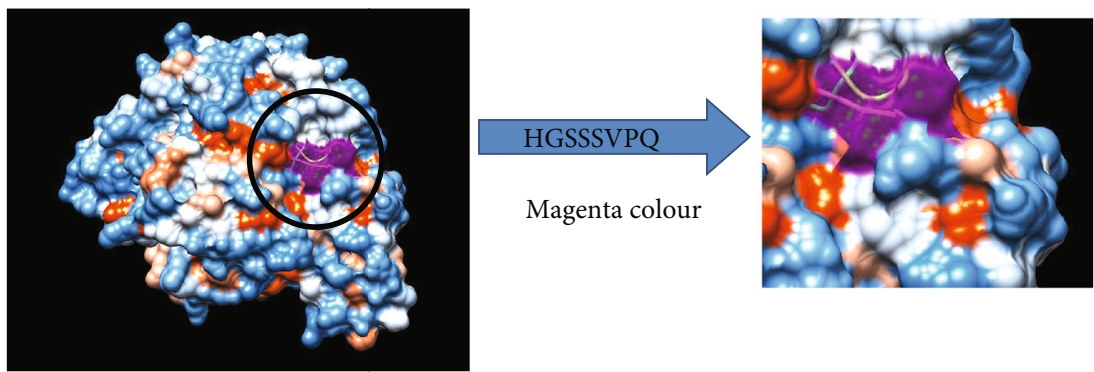

FIGURE 7: B cell epitopes proposed. The arrow shows the position of HGSSSVPQ with Magenta colour in a structural level of fructose 1,6bisphosphate aldolase. *The 3D structure was obtained using USCF Chimera software.

various $\mathrm{B}$ and $\mathrm{T}$ cell epitopes. Conserved epitopes would be considered candidate epitopes for B and T cell [23].

2.4. B Cell Epitope Prediction. B cell epitope is the portion of the vaccine that interacts with $B$ lymphocytes which are a type of white blood cell of the lymphocyte subtype. Candidate epitopes were analyzed using several $\mathrm{B}$ cell prediction methods from the IEDB (http://tools.iedb.org/bcell/) to identify the surface accessibility, antigenicity, and hydrophilicity with the aid of random forest algorithm, a form of unsupervised learning. The BepiPred linear prediction 2 was used to predict linear B cell epitope with the default threshold value 0.533 (http://tools.iedb.org/bcell/result/). The Emini surface accessibility prediction tool was used to detect the surface accessibility with the default threshold value 1.00 (http:// tools.iedb.org/bcell/result/). The Kolaskar and Tongaonkar antigenicity method was used to identify the antigenicity sites of a candidate epitope with the default threshold value 1.032 (http://tools.iedb.org/bcell/result/). The Parker hydrophilicity prediction tool was used to identify the hydrophilic, accessible, or mobile regions with the default threshold value 1.695 [24-28].

2.5. T Cell Epitope Prediction MHC Class I Binding. T cell epitope is the portion of the vaccine that interacts with T lymphocytes. Analysis of peptide binding to the MHC (major histocompatibility complex) class I molecule was assessed by the IEDB MHC I prediction tool (http://tools.iedb.org/mhci/ ) to predict cytotoxic $\mathrm{T}$ cell epitopes (also known as CD8+ cell). The presentation of peptide complex to T lymphocyte undergoes several steps. The Artificial Neural Network (ANN) 4.0 prediction method was used to predict the binding affinity. Before the prediction, all human allele lengths were selected and set to 9 amino acids. The half-maximal inhibitory concentration (IC50) value required for all conserved epitopes to bind was a score less than 500 [29-35].

2.6. T Cell Epitope Prediction MHC Class II Binding. Prediction of $\mathrm{T}$ cell epitopes interacting with MHC class II was assessed by the IEDB MHC II prediction tool (http://tools .iedb.org/mhcii/) for helper T cell, which is known as CD4+ cell also. Human allele reference set was used to determine the interaction potentials of $\mathrm{T}$ cell epitopes and MHC class II allele (HLA DR, DP, and DQ). The NN-align method was used to predict the binding affinity. IC50 score values less than 100 were selected [36-39].

2.7. Population Coverage. The population coverage tool was selected to analyze the epitopes in the IEDB. This tool calculates the fraction of individuals predicted to respond to a given set of epitopes with known MHC restriction (http:// tools.iedb.org/population/iedbinput). The appropriate checkbox for calculation was checked based on MHC I, MHC II separately, and a combination of both [40].

2.8. Homology Modelling. The 3D structure was obtained using RaptorX (http://raptorx.uchicago.edu), i.e., a protein structure prediction server developed by Peng and Xu's group, excelling at 3D structure prediction for protein sequences without close homologs in the Protein Data Bank (PDB). USCF Chimera (version 1.8) was the program used for visualization and analysis of molecular structure of the promising epitopes (http://www.cgl.uscf.edu/chimera) [41, 42]. 
TABLE 3: The most promising T cell epitopes and their corresponding MHC I alleles.

\begin{tabular}{|c|c|}
\hline Peptide & MHC I alleles \\
\hline AADKTDSPV & HLA-C $* 05: 01$, HLA-C $* 03: 03$ \\
\hline AAIEEFPHI & HLA-A $* 02: 06$ \\
\hline AIGTSHGAY & HLA-A $* 30: 02$, HLA-B $* 15: 01$, HLA-A $* 29: 02$ \\
\hline ETYGVPVEE & HLA-A $* 68: 02$ \\
\hline FNVNNLEQM & HLA-C $* 12: 03$ \\
\hline GEIKETYGV & HLA-B $* 40: 02$, HLA-B $* 40: 01$ \\
\hline GELGCLGSL & HLA-B $* 40: 01$, HLA-B $* 40: 02$ \\
\hline GTSHGAYKF & HLA-A $* 29: 02$, HLA-A $* 32: 01$, HLA-B $* 58$ \\
\hline IAIGTSHGA & HLA-A $* 02: 06$ \\
\hline IEEFPHIPV & HLA-B $* 40: 01$ \\
\hline IQLGFSSVM & HLA-B $* 15: 01$, HLA-A $* 02: 06$, HLA-B $* 15: 02$ \\
\hline ISLEGMFQR & HLA-A $* 31: 01$, HLA-A $* 68: 01$, HLA-A $* 11: 01$ \\
\hline IVQASAGAR & HLA-A $* 31: 01$, HLA-A $* 68: 01$ \\
\hline KPISLEGMF & HLA-B $* 35: 01$, HLA $-B * 07: 02$ \\
\hline KVNIDTDLR & HLA-A $* 31: 01$ \\
\hline LAIAIGTSH & HLA-B $* 35: 01$, HLA-C $* 03: 03$ \\
\hline LVMHGSSSV & HLA-A $* 02: 06$, HLA-A $* 68: 02$, HLA-C $* 12: 03$, HLA-C $* 14: 02$, HLA-A $* 02: 01$ \\
\hline NVNNLEQMR & HLA-A $* 68: 01$ \\
\hline NVRVTQQTV & HLA-A $* 30: 01$ \\
\hline QMLDHAAEF & HLA-A $* 02: 06$, HLA-A $* 29: 02$, HLA-B $* 15: 01$, HLA-B $* 15: 02$, HLA-A $* 32: 01$ \\
\hline RKVNIDTDL & HLA-B $* 48: 01$ \\
\hline SIQLGFSSV & HLA-A $* 02: 06$ \\
\hline SLEGMFQRY & HLA-A $* 29: 02$, HLA-A $* 30: 02$ \\
\hline SPVIVQASA & HLA-B $* 07: 02$ \\
\hline VIVQASAGA & HLA-A $* 02: 06$ \\
\hline VPAFNVNNL & HLA-B $* 07: 02$ \\
\hline YGGEIKETY & HLA-C $* 12: 03$ \\
\hline YGVPVEEIV & HLA-C $* 12: 03$ \\
\hline
\end{tabular}
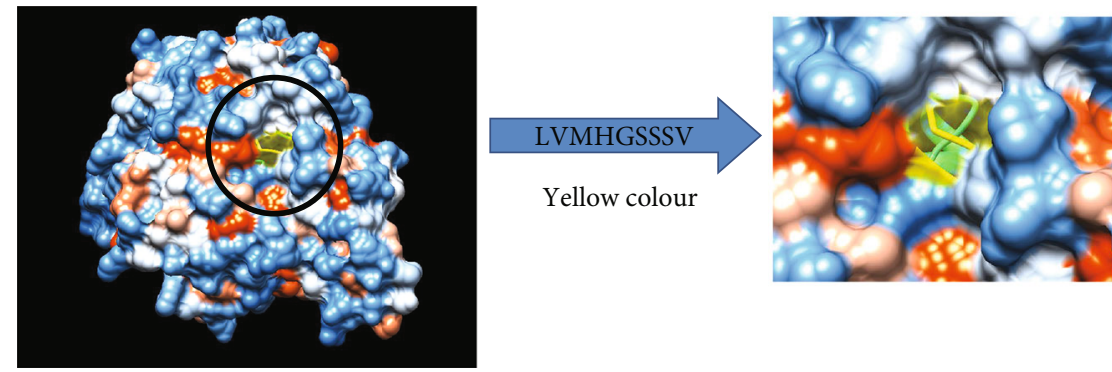

FIGURE 8: T cell epitopes proposed that interact with MHC I. The arrow shows the position of LVMHGSSSV with yellow colour in a structural level of fructose 1,6-bisphopsphate aldolase. ${ }^{*}$ The 3D structure was obtained using USCF Chimera software.

TABLE 4: The most promising T cell epitopes and their corresponding MHC II alleles.

\begin{tabular}{lc}
\hline Peptide & MHC II alleles \\
\hline KVNIDTDLRLASTGA & HLA-DRB1 $* 03: 01$, HLA-DRB $1 * 11: 01$ \\
GEIKETYGVPVEEIV & HLA-DRB $1 * 07: 01$, HLA-DRB $1 * 13: 02$, HLA-DQA $1 * 05: 01 / D Q B 1 * 02: 01$, \\
GGEIKETYGVPVEEI & HLA-DQA $1 * 04: 01 /$ DQB $1 * 04: 02$, HLA-DQA $* 03: 01 / D Q B 1 * 03: 02$ \\
& HLA-DRB $1 * 07: 01$, HLA-DRB $1 * 13: 02$, HLA-DQA $1 * 04: 01 /$ DQB $1 * 04: 02$ \\
YGGEIKETYGVPVEE & HLA-DQA $1 * 03: 01 /$ DQB $1 * 03: 02$ \\
\hline
\end{tabular}



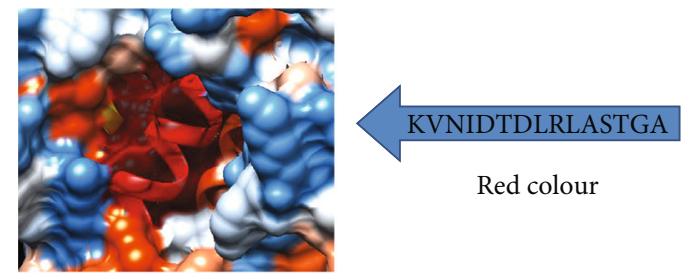

Red colour

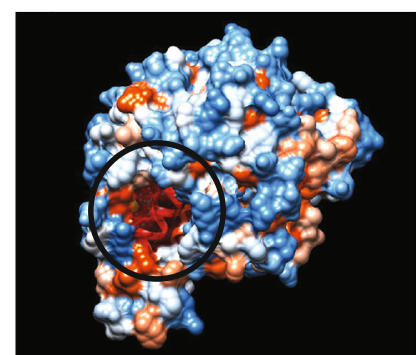

FIGURE 9: T cell epitopes proposed that interact with MHC II. The arrow shows the position of KVNIDTDLRLASTGA with red colour in a structural level of fructose 1,6-bisphosphate aldolase. *The 3D structure was obtained using USCF Chimera software.

TABLE 5: The population coverage of the whole world for the most promising epitopes of MHC I, MHC II, and MHC I and II combined.

\begin{tabular}{llcc}
\hline Country & MHC I & MHC II & MHC I,II (combined) \\
\hline World & $88.75 \%$ & $61.1 \%{ }^{*}$ & $95.62 \%{ }^{*}$ \\
\hline
\end{tabular}

*In the population coverage analysis of MHC II; 8 alleles were not included in the calculation; therefore, the above $\left({ }^{*}\right)$ percentages are for epitope sets excluding these alleles: HLA-DQA $*$ 05:01/DQB1 $* 03: 01$, HLA-DQA $* 01: 02 / \mathrm{DQB} 1 * 06: 02$, HLA-DQA1 $* 03: 01 / \mathrm{DQB} 1 * 03: 02$, HLA-DRB4 $* 01: 01$, HLA-DRB5 $* 01: 01$, HLA-DQA1 $* 05: 01 / \mathrm{DQB} 1 * 02: 01$, HLA-DPA1 $* 03: 01 / \mathrm{DPB} 1 * 04: 02$, HLA-DQA $1 * 04: 01 / \mathrm{DQB} 1 * 04: 02$.

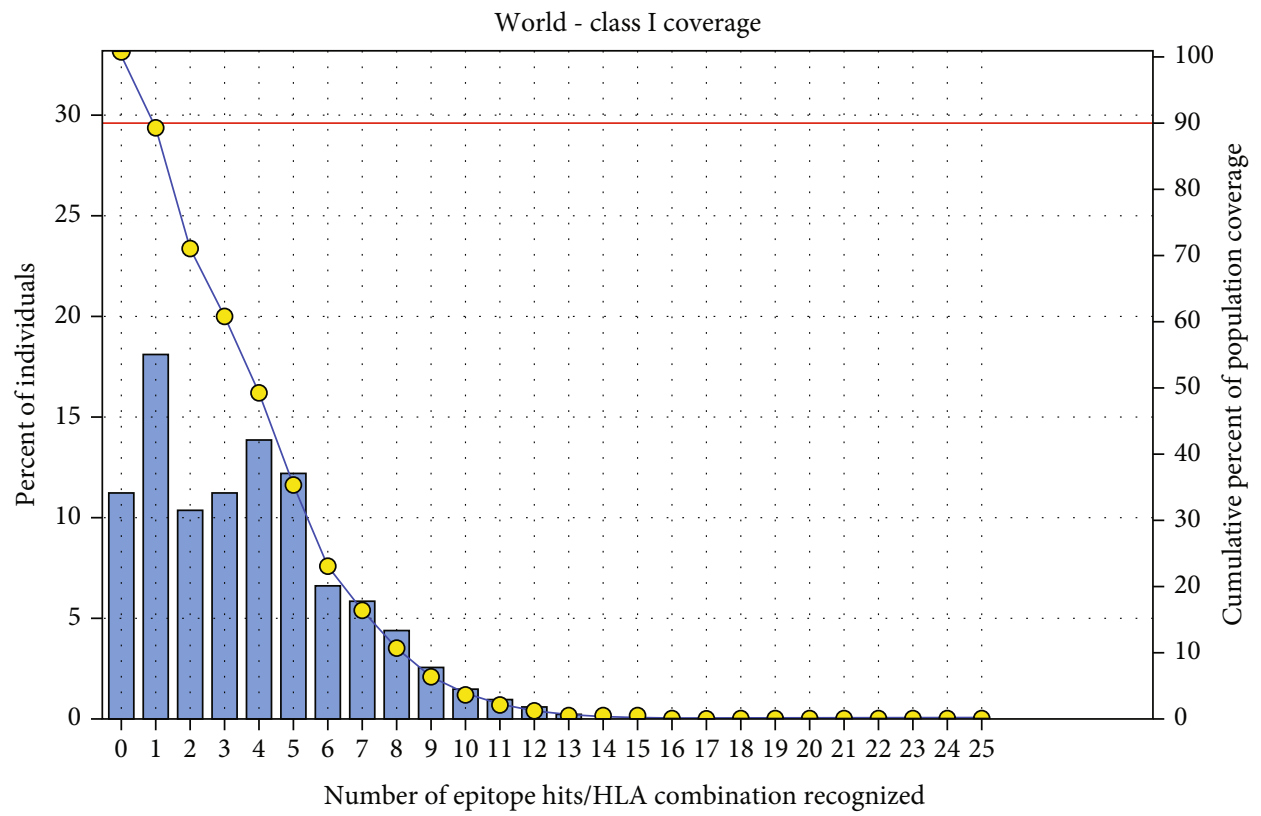

FIgURe 10: Population coverage for MHC class I epitopes.

\section{Results}

3.1. Amino Acid Composition. The amino acid composition for the reference sequence of Pseudomonas aeruginosa FBA is illustrated in Figure 1. Alanine and glycine were the most frequent amino acids (Table 1).

3.2. B Cell Epitope Prediction. The reference sequence of fructose 1,6-bisphosphate aldolase was subjected to BepiPred linear epitope prediction, Emini surface accessibility, Kolaskar and Tongaonkar antigenicity, and Parker hydrophilicity methods in the IEDB to test for various immunogenicity parameters (Table 2 and Figures 2-5). The tertiary structure of the proposed B cell epitopes is shown (Figures 6 and 7).
TABLE 6: Population coverage of the proposed peptide interaction with MHC class I.

\begin{tabular}{lcc}
\hline Epitope & Coverage (\%) & Total hits \\
\hline LVMHGSSSV & 60.41 & 7 \\
QMLDHAAEF & 31.70 & 8 \\
ISLEGMFQR & 25.64 & 3 \\
KPISLEGMF & 20.62 & 2 \\
LAIAIGTSH & 15.85 & 2 \\
\hline
\end{tabular}

3.3. Prediction of Cytotoxic T Lymphocyte Epitopes and Interaction with MHC Class I. The reference fructose 1,6bisphosphate aldolase sequence was analyzed using the (IEDB) MHC I binding prediction tool to predict T cell 


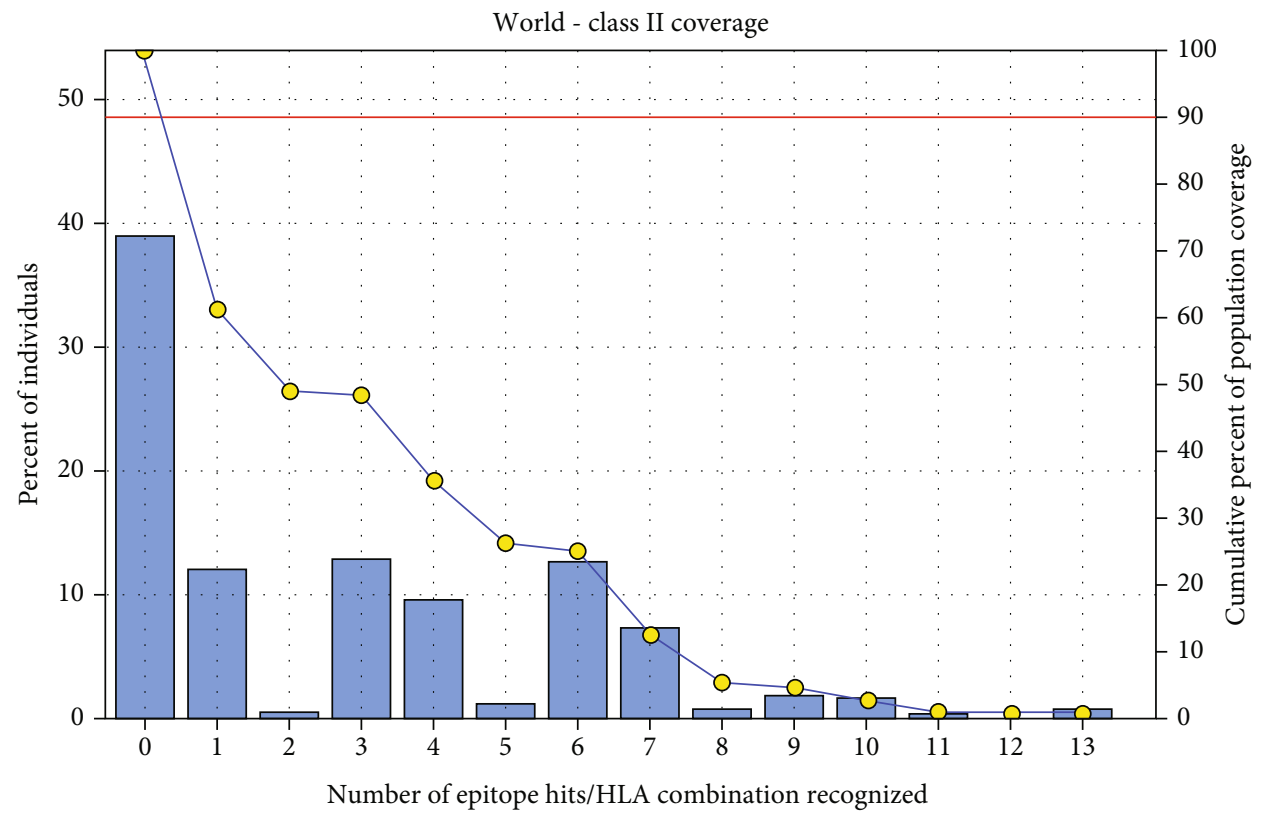

Figure 11: Population coverage for MHC class II epitopes.

epitopes which suggested interacting with different types of MHC class I alleles, based on Artificial Neural Network (ANN) with half-maximal inhibitory concentration (IC50) $<500 \mathrm{~nm}$. 206 peptides were predicted to interact with different MHC I alleles.

The most promising epitopes and their corresponding MHC I alleles are shown in Table 3 along with the 3D structure of the proposed one (Figure 8).

\subsection{Prediction of the T Cell Epitopes and Interaction with} MHC Class II. The reference fructose 1,6-bisphosphate aldolase sequence was analyzed using the (IEDB) MHC II binding prediction tool based on $\mathrm{NN}$-align with half-maximal inhibitory concentration (IC50) $<100 \mathrm{~nm}$; there were 662 predicted epitopes found to interact with MHC II alleles. The most promising epitopes and their corresponding alleles are shown in (Table 4) along with the 3D structure of the proposed one (Figure 9)

3.5. Population Coverage Analysis. All promising MHC I and MHC II epitopes of fructose 1,6-bisphosphate aldolase were assessed for population coverage against the whole world (Table 5).

For MHC I, epitopes with the highest population coverage were LVMHGSSSV (60.41\%) and QMLDHAAEF $(31.7 \%)$ (Figure 10 and Table 6). For MHC class II, the epitopes that showed the highest population coverage were KVNIDTDLRLASTGA (27.37\%) and GEIKETYGVPVEEIV, GGEIKETYGVPVEEI, and YGGEIKETYGVPVEE (24.27\%) (Figure 11 and Table 7). When combined together, the epitopes that showed the highest population coverage were LVMHGSSSV (60.41\%), QMLDHAAEF (31.7\%), and KVNIDTDLRLASTGA (27.37\%) (Figure 12).
TABLE 7: Population coverage of proposed peptides interaction with MHC class II.

\begin{tabular}{lcc}
\hline Epitope & Coverage (\%) & Total hits \\
\hline KVNIDTDLRLASTGA & $27.37 \%$ & 2 \\
GEIKETYGVPVEEIV & $24.27 \%$ & 5 \\
GGEIKETYGVPVEEI & $24.27 \%$ & 4 \\
YGGEIKETYGVPVEE & $24.27 \%$ & 2 \\
GVRKVNIDTDLRLAS & $23.90 \%$ & 2 \\
\hline
\end{tabular}

\section{Discussion}

Vaccination against $P$. aeruginosa is highly accredited due to the high mortality rates associated with the pathogen that spreads through healthcare areas. In addition, multidrug resistance of the pathogen demands the design of vaccine as an alternative [43]. In this study, immunoinformatics approaches were used to propose different peptides against FBA of P. aeruginosa for the first time. These peptides can be recognized by $\mathrm{B}$ cell and $\mathrm{T}$ cell to produce antibodies. Peptide vaccines overcome the side effects of conventional vaccines through easy production, effective stimulation of immune response, less allergy, and no potential infection possibilities [35]. Thus, the combination of humoural and cellular immunity is more promising at clearing bacterial infections than humoural or cellular immunity alone.

As B cells play a critical role in adaptive immunity, the reference sequence of $P$. Aeruginosa FBA was subjected to BepiPred linear epitope prediction 2 test to determine the binding to B cell, Emini surface accessibility test to test the surface accessibility, Kolaskar and Tongaonkar antigenicity test for antigenicity, and Parker hydrophilicity test for the hydrophilicity of the B cell epitope.

Out of the thirteen predicted epitopes using BepiPred 2 test, only three epitopes passed the other three tests 


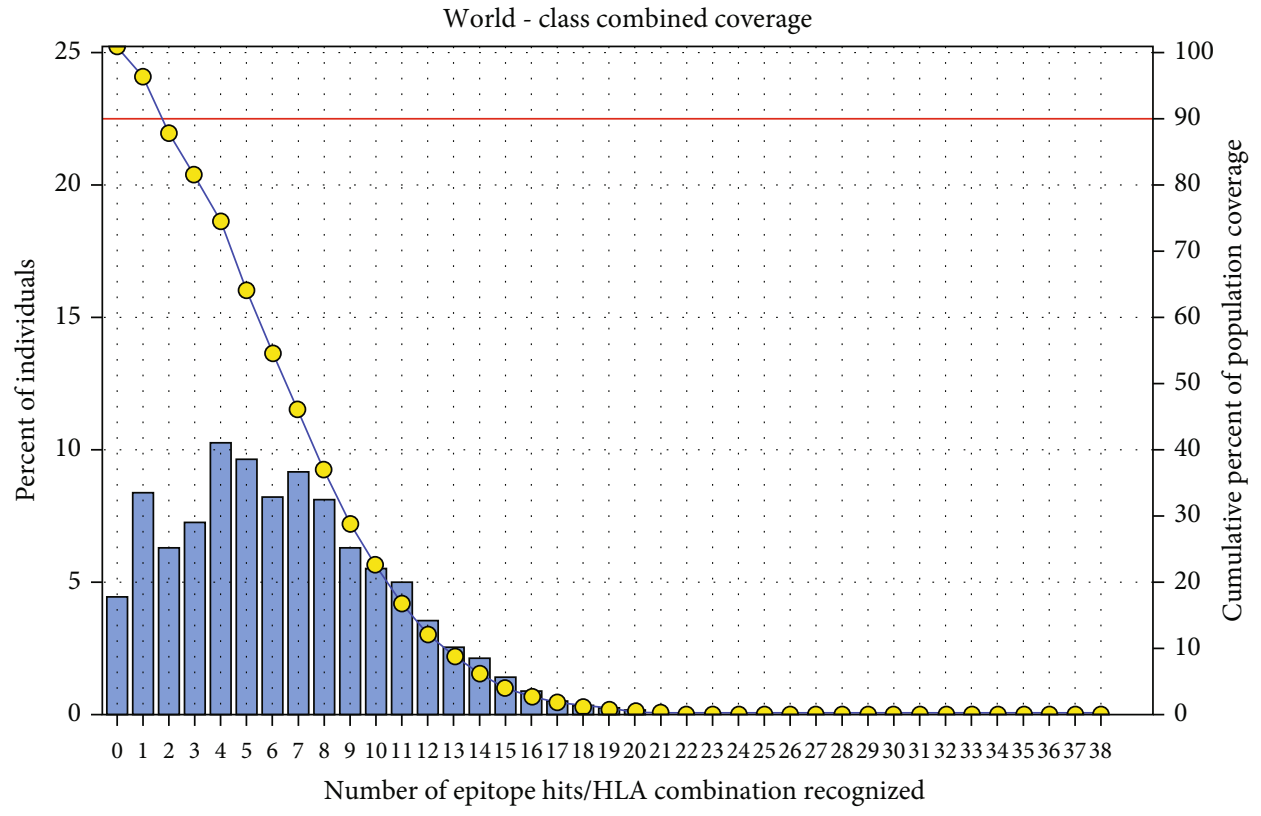

FIgURE 12: Population coverage for MHC class I and II epitopes combined.

(ADKTDSPVI, YNVRVTQQTV, and HGSSSVPQ) after segmentation. BepiPred version 2 test was used because it implements random forest and therefore predicts large epitope segments.

The reference sequence was analyzed using the IEDB MHC I and II binding prediction tools to predict T cell epitopes. 28 epitopes were predicted to interact with MHC I alleles with half-maximal inhibitory concentration $($ IC50) $<500$. Six of them were most promising and had the affinity to bind to the highest number of MHC I alleles (LVMHGSSSV, QMLDHAAEF, AIGTSHGAY, GTSHGAYKF, IQLGFSSVM, and ISLEGMFQR). 19 predicted epitopes were interacted with MHC II alleles with IC50 < 100. Four of them were most promising and had the affinity to bind to the highest number of MHC II alleles (GEIKETYGVPVEEIV, GGEIKETYGVPVEEI, KVNIDTDLRLASTGA, and YGGEIKETYGVPVEE). Nineteen epitopes (NVNNLEQMR, IQLGFSSVM, AADKTDSPV, SIQLGFSSV, GEIKETYGV, AIGTSHGAY, VPAFNVNNL, KVNIDTDLR, LAIAIGTSH, IVQASAGAR, ETYGVPVEE, GTSHGAYKF, YGGEIKETY, VIVQASAGA, IAIGTSHGA, RKVNIDTDL, FNVNNLEQM, YGVPVEEIV, and SPVIVQASA) appeared in both MHC I and II results.

The best epitope with the highest population coverage for MHC I was LVMHGSSSV (60.41\%) with seven HLA hits, and the coverage of population set for the whole MHC I epitopes was $88.75 \%$. Excluding certain alleles for MHC II, the best epitope was KVNIDTDLRLASTGA scoring $27.37 \%$ with two HLA hits, followed by GEIKETYGVPVEEIV scoring $24.27 \%$ with five HLA hits. The population coverage was $61.1 \%$ for all conserved MHC II epitopes. These epitopes have the ability to induce $\mathrm{T}$ cell immune response when interacting strongly with MHC I and MHC II alleles effectively generating cellular and humoural immune response against the invading pathogen. When combined, the epitope LVMHGSSSV had the highest population coverage percent $60.41 \%$ with seven HLA hits for both MHC I and MHC II.
Many studies had predicted peptide vaccines for different microorganisms such as rubella, Ebola, dengue, Zika, HPV, Lagos rabies virus, and mycetoma using immunoinformatics tools [44-51]. Limitations include the exclusion of certain HLA alleles for MHC II.

We hope that the world will benefit from these predicted epitopes in the formulation of the peptide-based vaccine and recommend further in vivo and in vitro studies to prove its effectiveness along with formulation of appropriate adjuvants. Finding another immunogenic target and analyzing the associated epitopes support the vaccine formula.

\section{Conclusion}

Vaccination is used to protect and minimize the possibility of infection leading to an increased life expectancy. The design of vaccines using immunoinformatics prediction methods is highly appreciated due to the significant reduction in cost, time, effort, and resources. Epitope-based vaccines are expected to be more immunogenic and less allergenic than traditional biochemical vaccines. We have illustrated different epitopes that have the ability to stimulate both $\mathrm{B}$ and $\mathrm{T}$ cells against fructose bisphosphate aldolase protein of $\mathrm{Pseu}$ domonas aeruginosa for the first time. Three B cell epitopes have successfully passed the required tests. Six MHC I epitopes were found to be most promising, while four were found from MHC II epitope result set. These epitopes covered $95.62 \%$ worldwide excluding certain MHC II alleles.

\section{Data Availability}

The data which support our findings in this study are available from the corresponding author upon reasonable request. 


\section{Conflicts of Interest}

The authors declare that there is no conflict of interest.

\section{Acknowledgments}

Many thanks go to the National Center for Biotechnology Information (NCBI), BioEdit, Immune Epitope Database (IEDB) Analysis Resource, RaptorX server, and UCSF Chimera team.

\section{References}

[1] K. N. Schurek, E. B. M. Breidenstein, and R. E. W. Hancock, "Pseudomonas aeruginosa: a persistent pathogen in cystic fibrosis and hospital-associated infections," Antibiotic Discovery and Development, pp. 679-715, 2012.

[2] B. J. Williams, J. Dehnbostel, and T. S. Blackwell, "Pseudomonas aeruginosa: host defence in lung diseases," Respirology, vol. 15, no. 7, pp. 1037-1056, 2010.

[3] S. L. Gellatly and R. E. W. Hancock, "Pseudomonas aeruginosa: new insights into pathogenesis and host defenses," Pathogens and Disease, vol. 67, no. 3, pp. 159-173, 2013.

[4] J. Chastre and J.-Y. Fagon, "Ventilator-associated pneumonia," American Journal of Respiratory and Critical Care Medicine, vol. 165, no. 7, pp. 867-903, 2002.

[5] H. W. Boucher, G. H. Talbot, J. S. Bradley et al., "Bad bugs, no drugs: no ESKAPE! An update from the Infectious Diseases Society of America," Clinical Infectious Diseases, vol. 48, no. 1, pp. 1-12, 2009.

[6] R. N. Jones, M. G. Stilwell, P. R. Rhomberg, and H. S. Sader, "Antipseudomonal activity of piperacillin/tazobactam: more than a decade of experience from the SENTRY Antimicrobial Surveillance Program (1997-2007)," Diagnostic Microbiology and Infectious Disease, vol. 65, no. 3, pp. 331-334, 2009.

[7] G. G. Zhanel, M. DeCorby, H. Adam et al., "Prevalence of antimicrobial-resistant pathogens in Canadian hospitals: results of the Canadian Ward Surveillance Study (CANWARD 2008)," Antimicrobial Agents and Chemotherapy, vol. 54, no. 11, pp. 4684-4693, 2010.

[8] P. Mahar, A. A. Padiglione, H. Cleland, E. Paul, M. Hinrichs, and J. Wasiak, "Pseudomonas aeruginosa bacteraemia in burns patients: risk factors and outcomes," Burns, vol. 36, no. 8, pp. 1228-1233, 2010.

[9] M.-L. Lambert, C. Suetens, A. Savey et al., "Clinical outcomes of health-care-associated infections and antimicrobial resistance in patients admitted to European intensive-care units: a cohort study," The Lancet Infectious Diseases, vol. 11, no. 1, pp. 30-38, 2011.

[10] K. Poole, "Pseudomonas aeruginosa: resistance to the max," Frontiers in Microbiology, vol. 2, p. 65, 2011.

[11] A. J. Kallen, A. I. Hidron, J. Patel, and A. Srinivasan, "Multidrug resistance among gram-negative pathogens that caused healthcare-associated infections reported to the National Healthcare Safety Network, 2006-2008," Infection Control \& Hospital Epidemiology, vol. 31, no. 5, pp. 528-531, 2010.

[12] E. F. Keen III, B. J. Robinson, D. R. Hospenthal et al., "Prevalence of multidrug-resistant organisms recovered at a military burn center," Burns, vol. 36, no. 6, pp. 819-825, 2010.

[13] E. B. Hirsch and V. H. Tam, "Impact of multidrug-resistant Pseudomonas aeruginosa infection on patient outcomes,"
Expert Review of Pharmacoeconomics \& Outcomes Research, vol. 10, no. 4, pp. 441-451, 2014.

[14] A. S. Ginsburg and K. P. Klugman, "Vaccination to reduce antimicrobial resistance," The Lancet Global Health, vol. 5, no. 12, pp. e1176-e1177, 2017.

[15] G. Döring and G. B. Pier, "Vaccines and immunotherapy against Pseudomonas aeruginosa," Vaccine, vol. 26, no. 8, pp. 1011-1024, 2008.

[16] M. I. Rashid, A. Naz, A. Ali, and S. Andleeb, "Prediction of vaccine candidates against Pseudomonas aeruginosa: an integrated genomics and proteomics approach," Genomics, vol. 109, no. 3-4, pp. 274-283, 2017.

[17] A. Sharma, A. Krause, and S. Worgall, "Recent developments for Pseudomonas vaccines," Human Vaccines, vol. 7, no. 10, pp. 999-1011, 2014.

[18] L. Florea, B. Halldorsson, O. Kohlbacher, R. Schwartz, S. Hoffman, and S. Istrail, "Epitope prediction algorithms for peptide-based vaccine design," in Computational Systems Bioinformatics. CSB2003. Proceedings of the 2003 IEEE Bioinformatics Conference. CSB2003.

[19] K. Prabhavathy, P. Perumal, and N. SundaraBaalaji, In silico identification of B-and T-cell epitopes on OMPLA and LsrC from Salmonella typhi for peptide-based subunit vaccine design, NISCAIR-CSIR, India, 2011.

[20] T. A. Ahmad, A. E. Eweida, and S. A. Sheweita, "B-cell epitope mapping for the design of vaccines and effective diagnostics," Trials in Vaccinology, vol. 5, pp. 71-83, 2016.

[21] T. Hall, I. Biosciences, and C. Carlsbad, "BioEdit: an important software for molecular biology," GERF Bulletin of Biosciences, vol. 2, no. 1, pp. 60-61, 2011.

[22] G. Zhang and B. Fang, "A uniform design-based back propagation neural network model for amino acid composition and optimal pH in G/11 xylanase," Journal of Chemical Technology \& Biotechnology, vol. 81, no. 7, pp. 1185-1189, 2006.

[23] R. Vita, S. Mahajan, J. A. Overton et al., "The immune epitope database (IEDB): 2018 update," Nucleic Acids Research, vol. 47, no. D1, pp. D339-D343, 2019.

[24] T. A. Hall, "BioEdit: a user-friendly biological sequence alignment editor and analysis program for Windows 95/98/NT," in Nucleic acids symposium series, pp. c1979-c2000, Information Retrieval Ltd, London, 1999.

[25] J. Larsen, O. Lund, and M. Nielsen, "Improved method for predicting linear B-cell epitopes," Immunome Research, vol. 2, no. 1, p. 2, 2006.

[26] E. A. Emini, J. V. Hughes, D. S. Perlow, and J. Boger, "Induction of hepatitis a virus-neutralizing antibody by a virusspecific synthetic peptide," Journal of Virology, vol. 55, no. 3, pp. 836-839, 1985.

[27] A. S. Kolaskar and P. C. Tongaonkar, "A semi-empirical method for prediction of antigenic determinants on protein antigens," FEBS Letters, vol. 276, no. 1-2, pp. 172-174, 1990.

[28] J. M. R. Parker, D. Guo, and R. S. Hodges, "New hydrophilicity scale derived from high-performance liquid chromatography peptide retention data: correlation of predicted surface residues with antigenicity and X-ray-derived accessible sites," Biochemistry, vol. 25, no. 19, pp. 5425-5432, 2002.

[29] M. Andreatta and M. Nielsen, "Gapped sequence alignment using artificial neural networks: application to the MHC class I system," Bioinformatics, vol. 32, no. 4, pp. 511-517, 2016.

[30] S. Buus, S. L. Lauemøller, P. Worning et al., "Sensitive quantitative predictions of peptide-MHC binding by a 'Query by 
Committee' artificial neural network approach," Tissue Antigens, vol. 62, no. 5, pp. 378-384, 2003.

[31] M. Nielsen, C. Lundegaard, P. Worning et al., "Reliable prediction of T-cell epitopes using neural networks with novel sequence representations," Protein Science, vol. 12, no. 5, pp. 1007-1017, 2003.

[32] B. Peters and A. Sette, "Generating quantitative models describing the sequence specificity of biological processes with the stabilized matrix method," BMC Bioinformatics, vol. 6, no. 1, p. 132, 2005.

[33] C. Lundegaard, K. Lamberth, M. Harndahl, S. Buus, O. Lund, and M. Nielsen, "NetMHC-3.0: accurate web accessible predictions of human, mouse and monkey MHC class I affinities for peptides of length 8-11," Nucleic acids research, vol. 36, supplemenr 2, pp. W509-W512, 2008.

[34] M. Abdelbagi, T. Hassan, M. Shihabeldin et al., "Immunoinformatics prediction of peptide-based vaccine against African horse sickness virus," Immunome Research, vol. 13, no. 2, p. 2, 2017.

[35] A. Patronov and I. Doytchinova, "T-cell epitope vaccine design by immunoinformatics," Open Biology, vol. 3, no. 1, p. 120139, 2013.

[36] P. Wang, J. Sidney, C. Dow, B. Mothé, A. Sette, and B. Peters, "A systematic assessment of MHC class II peptide binding predictions and evaluation of a consensus approach," PLoS Computational Biology, vol. 4, no. 4, article e1000048, 2008.

[37] P. Wang, J. Sidney, Y. Kim et al., "Peptide binding predictions for HLA DR, DP and DQ molecules," BMC Bioinformatics, vol. 11, no. 1, p. 568, 2010.

[38] Y. Kim, J. Ponomarenko, Z. Zhu et al., "Immune epitope database analysis resource," Nucleic Acids Research, vol. 40, no. W1, pp. W525-W530, 2012.

[39] M. Nielsen and O. Lund, "NN-align. An artificial neural network-based alignment algorithm for MHC class II peptide binding prediction," BMC bioinformatics, vol. 10, no. 1, p. 296, 2009.

[40] H.-H. Bui, J. Sidney, K. Dinh, S. Southwood, M. J. Newman, and A. Sette, "Predicting population coverage of T-cell epitope-based diagnostics and vaccines," BMC Bioinformatics, vol. 7, no. 1, p. 153, 2006.

[41] E. F. Pettersen, T. D. Goddard, C. C. Huang et al., "UCSF Chimera-a visualization system for exploratory research and analysis," Journal of Computational Chemistry, vol. 25, no. 13, pp. 1605-1612, 2004.

[42] J. Peng and J. Xu, "RaptorX: exploiting structure information for protein alignment by statistical inference," Proteins: Structure, Function, and Bioinformatics, vol. 79, no. S10, pp. 161171, 2011.

[43] J. W. Costerton, P. S. Stewart, and E. P. Greenberg, "Bacterial biofilms: a common cause of persistent infections," Science, vol. 284, no. 5418, pp. 1318-1322, 1999.

[44] A. A. Mohammed, A. M. H. ALnaby, S. M. Sabeel et al., "Epitope-based peptide vaccine against fructose-bisphosphate aldolase of Madurella mycetomatis Using immunoinformatics approaches," Bioinformatics and Biology Insights, vol. 12, article 117793221880970, 2018.

[45] S. N. E. M. Elgenaid, E. M. Al Hajj, A. A. Ibrahim et al., "Prediction of multiple peptide based vaccine from E1, E2 and capsid proteins of rubella virus: an in-silico approach," Immunome Research, vol. 14, no. 1, p. 2, 2018.
[46] S. M. Bolis, W. A. Omer, M. A. Abdelhamed et al., "Immunoinformatics prediction of epitope based peptide vaccine against Madurella mycetomatis translationally controlled tumor protein," bioRxiv, p. 441881, 2018.

[47] A. A. Mohammed, O. Hashim, E. KAA, A. Hamdi, and M. A. Hassan, "Epitope-based peptide vaccine design against Mokola rabies virus glycoprotein $G$ utilizing in silico approaches," Immunome Research, vol. 13, no. 3, p. 2, 2017.

[48] S. O. A. Albagi, O. H. Ahmed, M. A. Gumaa, K. A. A. Elrahman, A. H. A. Haraz, and A. Mohammed, "Immunoinformatics-peptide driven vaccine and in silico modeling for Duvenhage rabies virus glycoprotein G," Journal of Clinical \& Cellular Immunology, vol. 8, no. 4, p. 2, 2017.

[49] A. H. A. Haraz, K. A. A. Elrahman, M. S. Ibrahim et al., "Multi epitope peptide vaccine prediction against Sudan Ebola virus using immuno-informatics approaches," Advanced Techniques in Biology \& Medicine, vol. 5, no. 203, 2017.

[50] M. M. O. Sahar Suliman Mohamed, S. M. Sami, H. A. Elgailany et al., "Immunoinformatics approach for designing epitopebased peptides vaccine of L1 major capsid protein against HPV type 16," International Journal of Multidisciplinary and Current Research, 2016.

[51] M. M. B. Suluiman, M. M. Osman, A. A. E. F. Alla et al., "Highly conserved epitopes of Zika envelope glycoprotein may act as a novel peptide vaccine with high coverage: immunoinformatics approach," Journal of Vaccines \& Vaccination, vol. 4 , no. 3, pp. 46-60, 2016. 